\title{
CREACIÓN DE UNA BASE DE DATOS HOMOGENEIZADA DE TEMPERATURAS PARA LOS PIRINEOS (1950-2010)
}

\author{
José M. Cuadrat, Roberto Serrano, Miguel A. Saz y Ernesto Tejedor \\ Departamento de Geografía. Universidad de Zaragoza \\ jmcuadrat@unizar.es \\ Marc Prohom y Jordi Cunillera \\ Àrea de Climatologia. Servei Meteorològic de Catalunya \\ Pere Esteban \\ Centre d'Estudis de la Neu i la Muntanya d'Andorra \\ Jean-Michel Soubeyroux y Nahalie Deaux \\ Direction de la Climatologie. Meteo France
}

\begin{abstract}
Resumen: En este artículo se describe el proceso y metodología para la creación de una base de datos térmica del Pirineo para el periodo temporal 1950-2010, y se avanzan los primeros resultados del análisis espacial de las temperaturas y del cálculo de la tendencia de las mismas a escala anual. Para el control de calidad y homogeneización se ha empleado el método HOMER que incorpora los últimos métodos y técnicas de gestión de datos, incluyendo la eliminación de valores atípicos, la detección por pares y la selección de punto de ruptura (reforzada por los metadatos). Las series finales proporcionan datos homogeneizados para analizar la evolución del clima y el comportamiento climático del conjunto del Pirineo.
\end{abstract}

Palabras clave: temperatura, bases de datos, homogeneización, Pirineos.

Abstract: In this work we focused on create a climate database of 66 monthly temperature series in the period 1950-2010. Each series has been quality controlled and homogenized by HOMER script, which incorporates the latest methods and techniques on data management, including outlier removing, pairwise detection, and breakpoint selection (enhanced by

Recibido: 29-11-13. Aceptado: 12-12-13. 
metadata). To evaluate the quality of initial series through the homogenization process is used the MDA (Minimum Detectable Amplitude) value. The final series provide homogenized data to analyze the evolution of the climate and climatic behaviour in the Pyrenees.

Key words: temperature database, homogeneity, Pyrenees.

\section{Introducción}

La disponibilidad de bases de datos climáticas de alta calidad es esencial para el estudio del clima y en particular para analizar su variabilidad y tendencia, dentro del actual contexto de preocupación por el cambio climático y sus impactos sobre las actividades humanas. Desafortunadamente, las observaciones meteorológicas están afectadas con frecuencia por circunstancias ajenas al comportamiento del clima, tales como variaciones de emplazamiento, cambios de instrumentación, alteración del entorno de los observatorios, etc.; por este motivo, para la obtención de una base de datos de calidad, las series climáticas deben someterse a un proceso de análisis de homogeneidad para que cualquier cambio o tendencia registrada sea respuesta directa de la evolución del clima y no de aspectos externos no climáticos.

Para abordar este problema se han desarrollado diversos métodos de homogeneización que intentan discriminar la señal propiamente climática del ruido producido por los factores mencionados. La mayor parte de la metodología empleada se basa en comparar las series problema con otras supuestamente homogéneas o bien construirlas sintéticamente a partir de otras series vecinas con el objetivo de diluir las inhomogeneidades en la serie compuesta (los trabajos de Peterson et al., 1998, y Aguilar et al., 2003, ofrecen una buena síntesis de estas metodologías). De esta forma se han confeccionado bases de datos de gran calidad y longitud temporal de ámbito mundial (Mitchell y Jones, 2005; Beck et al., 2005), continental, especialmente en Europa (Klok y Klein Tank, 2009), y nacional (Brunetti et al., 2006; Begert et al., 2005; González Hidalgo et al., 2011), pero en todos los casos se hace evidente la escasa representación de las áreas de montaña a causa de la dificultad de disponer de largas series de datos y el limitado número de observatorios. En este sentido, en Europa es destacable el ejemplo de la cordillera de los Alpes donde el proyecto HISTALP trabaja desde hace años en la recuperación de series climáticas (Böhm et al., 2009), y el proyecto CARPATCLIM, en los Cárpatos, en el que participan los diferentes países de la región (JCR, 2010).

En los Pirineos muchos proyectos han iniciado trabajos de recuperación y control de datos (Soubeyroux et al., 2011; Esteban, et al., 2012; Espejo et al., 2008), pero estas series climáticas son parciales y de calidad muy variable; además, están limitadas por los enfoques estrictamente nacionales, reduciendo la capacidad de análisis global de 
las variaciones climáticas. Desde esta perspectiva, el Observatorio Pirenaico del Cambio Climático tiene como objetivo la creación de una base de datos única de temperaturas y precipitaciones, siguiendo una metodología común de control de calidad y homogeneización, que facilite la elaboración de indicadores climáticos y permitan realizar un diagnóstico completo de las tendencias observadas. El trabajo se desarrolla de forma coordinada entre Meteo France, la Agencia Estatal de Meteorología, el Servei Meteorològic de Catalunya, el Centre d’Estudis de la Neu i la Muntanya d'Andorra y la Universidad de Zaragoza. En este artículo se describe el proceso y metodología para la construcción de la base de datos térmica para el periodo temporal 1950-2010, y se avanzan los primeros resultados del análisis espacial de las temperaturas y del cálculo de la tendencia de las mismas a escala anual.

\section{Fuentes y metodología}

\subsection{La base de datos climática}

Para la creación de la base de datos se han utilizado las fuentes de información de diferentes organismos meteorológicos: Meteo-France, el Centre d'Estudis de la Neu i de la Muntanya d'Andorra (CENMA), el Servei Meteorològic de Catalunya y la Agencia Estatal de Meteorología (AEMET). Para los objetivos planteados, el periodo de estudio considerado ha sido el de 1950 a 2010, y se seleccionaron aquellas series de datos con esa cobertura temporal, o que al menos cubrieran ese período en un $75 \%$. En aquellos casos en los que ha sido necesario utilizar información procedente de dos o más puntos de observación para obtener una serie completa, los criterios seguidos para su uso han sido los siguientes: 1) que estuvieran a una distancia menor de $20 \mathrm{~km}$; 2) una diferencia de altura inferior a $50 \mathrm{~m}$; y 3) preferiblemente un máximo de tres series individuales para confeccionar una única serie final. Una vez realizada esta aproximación, la base de datos se ha confeccionado con un total de 66 series de temperaturas.

\subsection{El método de análisis HOMER}

El objetivo principal de la Acción COST ES0601: Advances in homogenisation methods of climate series: an integrated approach (HOME), de la Unión Europea, consistía en determinar una metodología estándar para el análisis de homogeneidad de bases de datos ambientales y climáticos. Fruto de esta acción, en 2011 se generó el método HOMER (ver Mestre et al., 2013) para la homogeneización de series mensuales y anuales de temperatura y precipitación, empleado en este trabajo.

HOMER incluye las mejores prestaciones de otros métodos ya existentes, tales como PRODIGE, ACMANT y cghseg, un método de segmentación conjunta desarrollado desa- 
rrollado inicialmente por bio-estadísticos en el campo de la segmentación del ADN (Picard et al., 2011). Los resultados de diversos experimentos a ciegas realizados durante la acción COST, validaron estas aproximaciones, ya que PRODIGE y ACMANT resultaron estar entre los mejores métodos para homogeneizar series climáticas de resolución anual y mensual (Venema et al. 2012). HOMER es un método interactivo y semi-automático, ya que durante su aplicación el usuario puede escoger entre la salida totalmente automática que le ofrece el resultado surgido de cghseg (detección) y la técnica de comparación por parejas, en parte subjetiva, que le ofrece la adaptación del método PRODIGE. Al mismo tiempo, incluyen algunas innovaciones adaptadas de ACMANT, y se incorporan diferentes técnicas de análisis de calidad de las series procedentes de otra metodología: CLIMATOL (Guijarro, 2011). Finalmente, también agrega la metodología UBRIS para la caracterización de posibles tendencias en la temperatura atribuibles al efecto de la urbanización.

\subsection{Control de calidad de las series}

El control de calidad de los datos es un aspecto crucial en cualquier base de datos climática. Los errores en una observación meteorológica pueden proceder de un amplio abanico de motivos, de los cuáles los más comunes son fallos instrumentales, errores del propio observador, errores en la transcripción de los datos o en la posterior digitalización. En este punto debe distinguirse entre errores puntuales, de corto alcance temporal (días o alguna semana) y errores de amplia influencia y que persisten en el tiempo (inhomogeneidades).

En este estudio, las series mensuales y anuales se han analizado a partir de una inspección visual de los datos que ofrece el programa HOMER. Mediante la contextualización de cada dato mensual de una serie con sus series vecinas y climáticamente próximas, pueden detectarse valores anómalos o fuera de rango climático (los conocidos como outliers). La figura 1 es un ejemplo de este análisis, en la que se puede identificar, al menos, tres valores claramente erróneos para la serie de temperatura mínima de Molinos, en el Pirineo catalán. Una vez detectados estos valores erróneos, y siempre que esto ha sido posible, se han consultado las fuentes originales, para confirmar el error y substituirlo por los valores correctos. Si esto no ha sido posible, el valor se ha reemplazado por un "no dato".

\subsection{Análisis de homogeneidad}

El trabajo del análisis de homogeneidad consiste en una sucesión de ciclos: un ciclo de detección, seguido de un ciclo de corrección o de ajuste, al que le sigue un nuevo ciclo de detección y corrección. Seguidamente, se muestran los criterios utilizados para la realización de este proceso. 

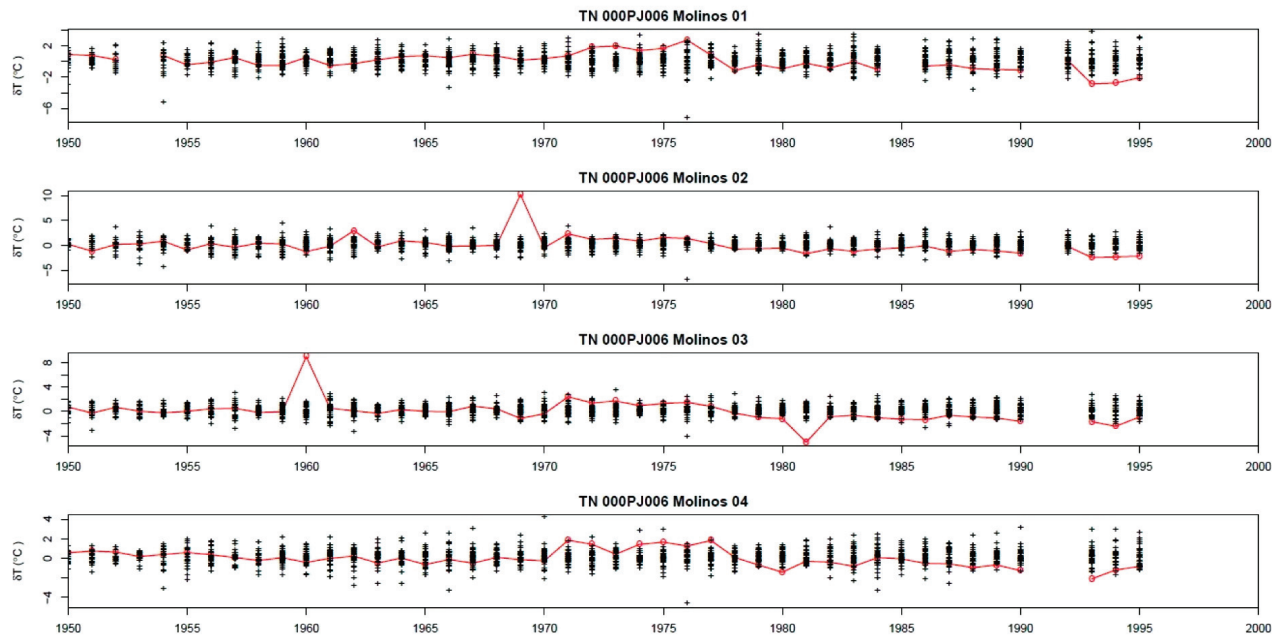

Figura 1. Salida gráfica del control de calidad aplicado a la serie de Molinos (Catalunya) en la que se aprecian tres valores fuera de rango: febrero de 1969, marzo de 1960 y marzo de 1981.

\section{a) Detección de los puntos de discontinuidad}

Con el apoyo de los metadatos disponibles, se ha realizado una aproximación estadística para detectar las discontinuidades en las series mensuales e identificar de manera más fiable la naturaleza de la discontinuidad. En todas las metodologías que intentan detectar los puntos de discontinuidad/ruptura, el principal reto es determinar si ese punto lo es realmente y no es fruto del azar o de un artificio estadístico. El método HOMER asume que cada uno de los valores de las series que forman el conjunto a homogeneizar puede ser descompuesto en un efecto climático, común a todas ellas, y un efecto de estación, constante en caso de serie homogénea, y variable en el tiempo en caso de ser inhomogénea. La aproximación inicial, por el método PRODIGE, comparaba las series de forma emparejada (pairwise) con el fin de detectar el número y posición de los puntos de cambio. HOMER, gracias a la incorporación del algoritmo cghseg, añade la posibilidad de realizar la detección simultánea en el conjunto de series y de manera automática.

A pesar del avance que supone el uso de HOMER, en cuanto a la sistematización y automatización del proceso de análisis de homogeneidad, existen todavía decisiones que deben ser definidas previamente, y que fueron las siguientes:

- En esta fase del análisis (detección de puntos de ruptura), se seleccionaron la totalidad de las series en cada subconjunto, es decir de ambas vertientes, para incluir series procedentes de estaciones meteorológicas gestionadas por diferentes 
organismos y evitar problemas de detección en casos de cambios masivos y coetáneos en parte o la totalidad de una misma red (Kuglitsch et al., 2012).

- Para la red seleccionada, y debido al elevado número de series, se dividió en dos grandes conjuntos, el occidental y el oriental, y con la incorporación de diversas series de solape en ambos grupos.

- Para determinar un punto de ruptura como válido, se consideraron como ciertos aquellos concentrados alrededor de un mismo año y en un mínimo de tres series vecinas pertenecientes a la misma subred, y todo ello contrastado con el metadato disponible.

La naturaleza semiautomática de HOMER permite cotejar los resultados que ofrece la salida subjetiva de la aproximación PRODIGE, con los de la detección conjunta que produce cghseg. La figura 2 muestra la salida gráfica de la comparación por parejas en la serie de temperatura de Bagnères-de-Luchon (se ofrecen sólo las seis primeras series de la comparativa). A simple vista es posible detectar un mínimo de dos cambios en 1964, 1977 y probablemente uno o dos más alrededor de 1995.

Una vez definido el primer listado de puntos de ruptura, se procede a la corrección o ajuste de las series, generándose la primera versión de series homogeneizadas. Con esta primera versión, ya puede ejecutarse la fase de detección mediante la aproximación ACMANT. A diferencia de PRODIGE, ACMANT es un proceso totalmente automático y requiere para su funcionamiento de series prehomogeneizadas (series de referencia). Asimismo, ACMANT es capaz de encontrar cambios con un fuerte comportamiento estacional en series de temperatura (sólo se aplica a este tipo de variable), es decir, aquellos casos en los que debido a cambios en las condiciones de observación, especialmente asociadas a la exposición de los instrumentos o a la tipología de los abrigos, pueden existir respuestas de signo opuesto en función de la época del año: signo positivo en verano, y negativo en invierno. Adicionalmente, ACMANT permite detectar el punto de cambio a una resolución mensual, si no se dispone de metadato.

Finalmente, una vez corregidas las series, es necesario repetir el proceso de detección, para discriminar posibles puntos de ruptura que no se habían considerado en la primera fase o descartar aquéllos que se habían seleccionado erróneamente. Esta fase utiliza la misma muestra de series de referencia que en la primera etapa.

\section{b) Ajuste de las series}

Una vez identificados los puntos potenciales de ruptura para cada serie, se procede al ajuste de las mismas. El ajuste de series mensuales se realiza mediante un modelo ANOVA de dos factores. La técnica ANOVA consiste en un análisis de la varianza y se utiliza cuando se quieren contrastar más de dos medias. La inclusión del modelo ANOVA para la corrección de las series no homogéneas mejora significativamente los resultados de otros métodos, entre ellos el Standard Normal Homogeneity Test (SNHT) (Do- 
mokos, 2013). La aproximación ANOVA permite también la restitución de meses o años sin datos. Al igual que en la fase de detección, en esta fase debemos decidir algunos aspectos previos antes de realizar la corrección de las series:

- Definición de la red de series de contorno para el ajuste de cada una de las series, mediante el criterio de la correlación. Así, para series de temperatura máxima (Tx) se sugiere el umbral mínimo de 0.8 y para un mínimo de 6 series que cumplan ese criterio, mientras que para la temperatura mínima (Tn) se fija el umbral en 0.75 . En aquellos casos en los que no exista ninguna serie vecina que cumpla el criterio de correlación, se descartará la serie.

- Al tratarse de series de temperatura, el criterio de ajuste será el aditivo.
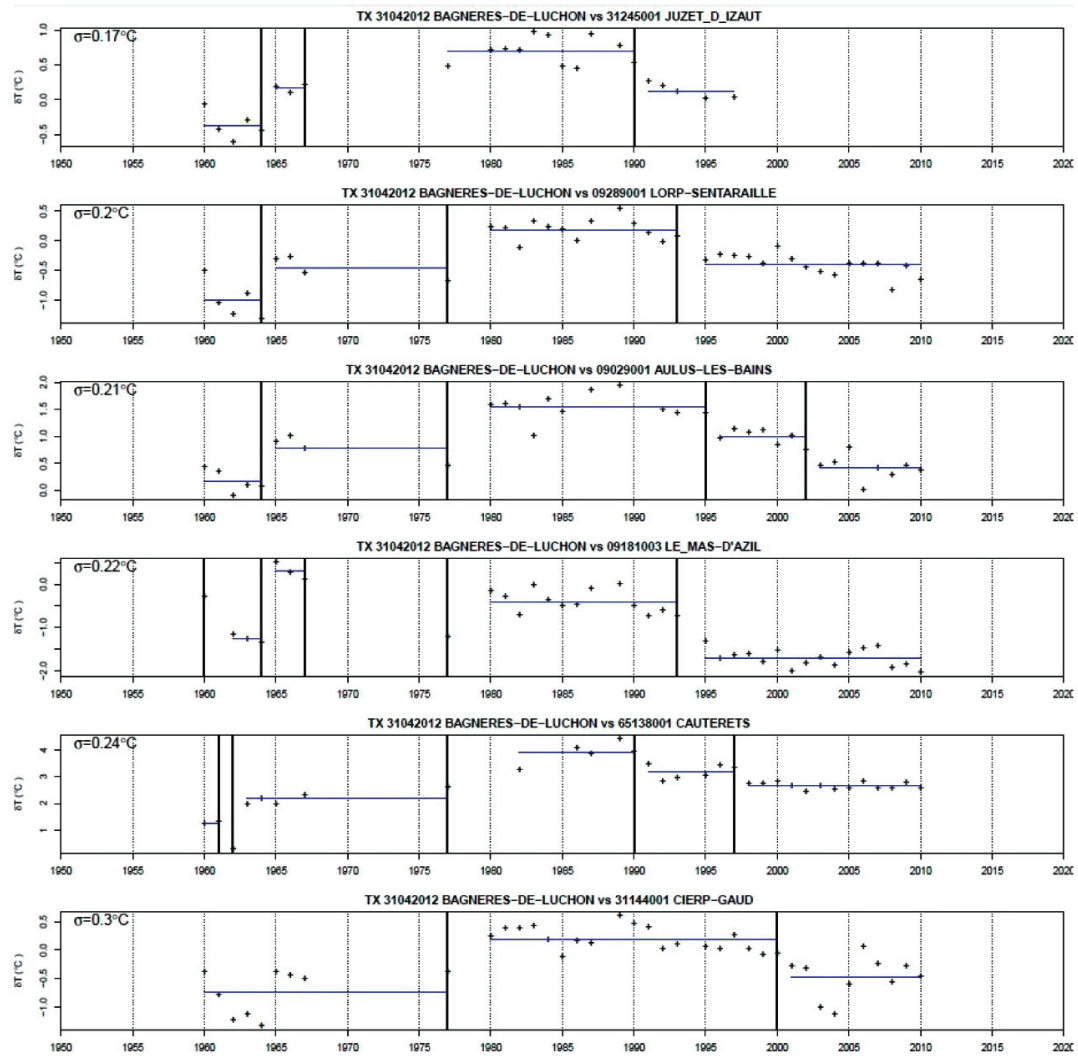

Figura 2. Salida gráfica de HOMER durante la fase de comparación por parejas: serie de temperatura máxima media anual de Bagnères-de-Luchon. La comparativa por parejas se ordena de arriba a abajo en función de los valores de la desviación estándar del ruido o

Amplitud Mínima Detectable (AMD). Cuanto más bajo es el valor, mayor será la capacidad de detección de puntos de ruptura. 


\section{Resultados y discusión}

El proceso seguido ha permitido identificar 57 series con una cobertura temporal mínima de 1959 a 2010 (de éstas, la mayor parte cubren el período 1950-2010), más otras 9 de apoyo y con una cobertura menor. La tabla 1 muestra la distribución altitudinal de las 57 series (de temperatura máxima y mínima) y su calificación de calidad, una vez aplicado el proceso de identificación y ajuste propio del análisis de homogeneidad.

De las 57 series, dos tercios ofrecen una calidad óptima para su uso en estudios climáticos: 63\% para las temperaturas máximas y 68\% para las temperaturas mínimas. De ellas, se identifican un total de 9 series de elevada calidad para las temperaturas máximas y únicamente 6 para las temperaturas mínimas. Por regla general, existen más series de buena o muy buena calidad en las series de temperatura máxima que en las de temperatura mínima, debido a la menor dependencia de la orografía accidentada de la región. De hecho, la frecuencia de series de calidad pobre aumenta con la altura y suelen ser de calidad buena o muy buena en series localizadas por debajo de los 500 metros.

Su distribución geográfica no es la óptima, puesto que amplias áreas del Pirineo axial quedan huérfanas de series de calidad aceptable, así como el ámbito más occidental de la vertiente sur, y especialmente para las series de temperatura máxima. Al mismo tiempo, aquellas series localizadas a altitudes superiores a los 1500 m muestran una calidad menor. Para minimizar esta incertidumbre, se requiere disponer de más observaciones en el ámbito del Pirineo, especialmente en aquellos sectores de mayor altitud, impulsar actuaciones de rescate de datos y asegurar la pervivencia de las observaciones ya existentes, así como de su buena calidad.

Tabla 1. Distribución de la calidad de las series por franjas altitudinales

\begin{tabular}{|l|c|c|c|c|c|}
\hline Altura $(\mathrm{m})$ & Núm. de series & Muy buena & Buena & Aceptable & Débil \\
\hline$<200$ & 13 & 3 & 7 & 3 & 0 \\
$200-500$ & 16 & 5 & 2 & 4 & 5 \\
$500-750$ & 13 & 2 & 3 & 4 & 4 \\
$750-1000$ & 10 & 0 & 1 & 1 & 8 \\
$1000-1500$ & 10 & 0 & 2 & 3 & 5 \\
$1500-2000$ & 3 & 0 & 2 & 1 & 0 \\
$>2000$ & 1 & 0 & 0 & 0 & 1 \\
\hline
\end{tabular}

La creación de esta base de datos única, con control de calidad y homogeneizada, permite realizar por primera vez una cartografía de la distribución de las temperaturas del conjunto del Pirineo y posibilita el examen comparativo de la vertiente francesa y espa- 
ñola, además de observar las significativas diferencias entre los distintos valles. El análisis preliminar de los datos muestran obviamente el natural descenso de los valores térmicos con la altitud, desde los 9-10 $\mathrm{C}$ de promedio anual del Prepirineo hasta los menos de $3^{\circ} \mathrm{C}$ que con probabilidad se alcanzan en las altas cumbres de la zona central.

Los resultados ponen en evidencia también las dificultades de estudio del clima de las áreas de montaña por la debilidad de la red de estaciones meteorológicas y la escasez de información disponible a partir de cierta altitud, que en el caso pirenaico se hace muy evidente desde los $1.500 \mathrm{~m}$. Este problema genera siempre notables incertidumbres en los cálculos que puedan hacerse para las altas cimas y la necesidad de tomarlos con mucha cautela.

Respecto a la evolución temporal de las temperaturas, el valor medio anual muestra un claro incremento a lo largo del periodo de estudio, calculado en torno a $0,2^{\circ} \mathrm{C}$ por década (figura 4). El aumento es más importante en las temperaturas máximas que en el promedio de las mínimas, y estacionalmente el incremento es mayor en las máximas estivales. Estos resultados son coincidentes con otros estudios similares de regiones vecinas y con la tendencia general del clima en Europa occidental.

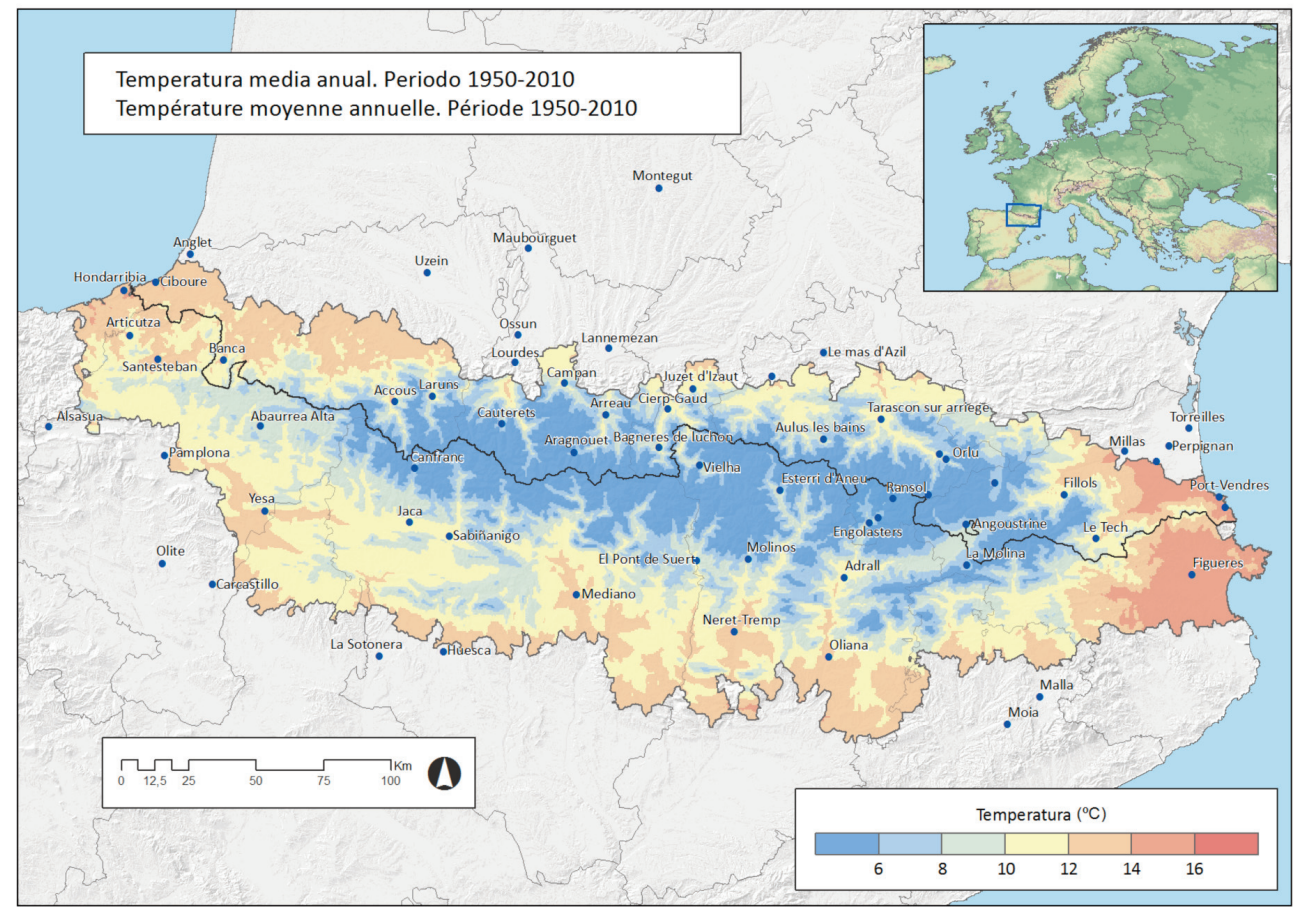

Figura 3. Distribución de la temperatura media anual en el Pirineo en el periodo 1950-2010. 


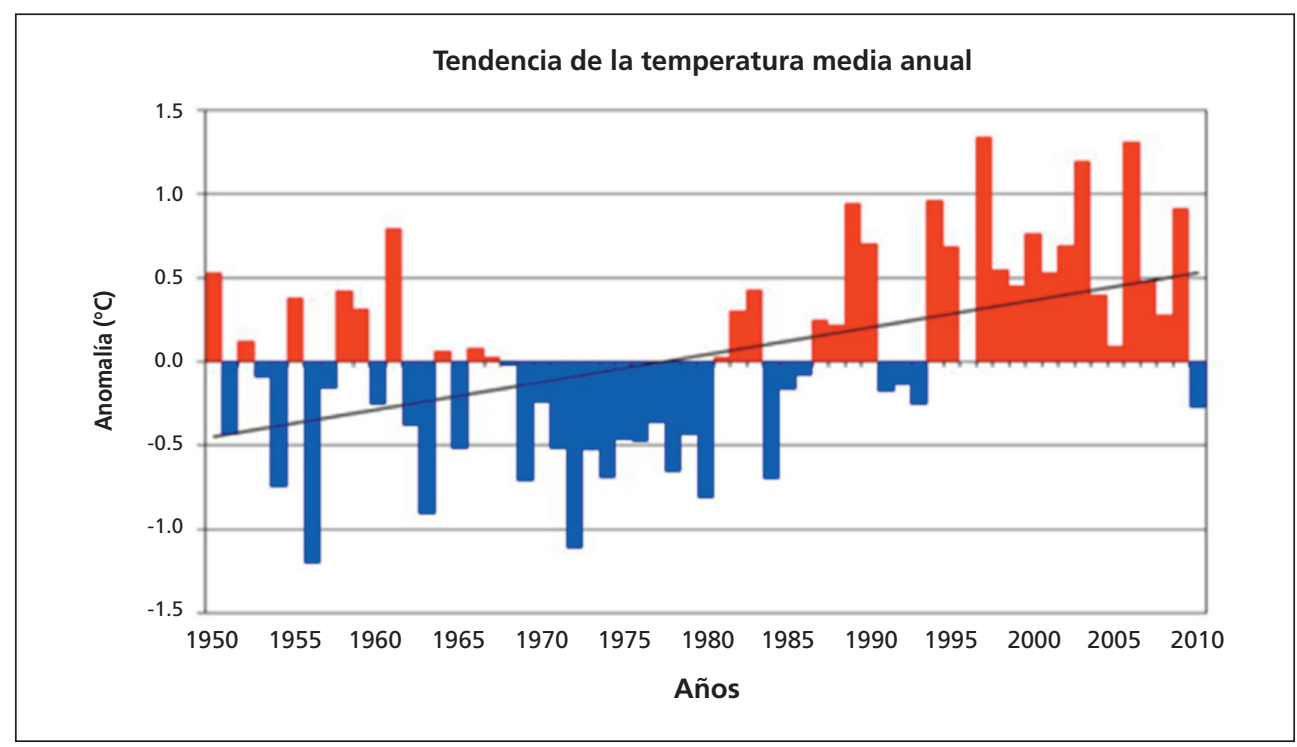

Figura 4. Anomalía de la temperatura media anual respecto al periodo 1971-2000 para el conjunto del Pirineo.

\section{Conclusiones}

El control de calidad y homogeneidad aplicado a las series de temperatura de la región pirenaica ha permitido crear una base de datos fiable y homogeneizada que posibilita un buen conocimiento de la distribución espacial de las temperaturas y la naturaleza de su tendencia reciente.

Fruto de este análisis de calidad, se han identificado aquellos valores erróneos o sospechosos en las series mensuales de temperatura máxima y mínima, substituyéndolos por su valor real, cuando esto era posible, o por valores no conocidos. Y al mismo tiempo, el contraste de homogeneidad ha permitido determinar los desajustes en las series, atribuibles a efectos no climáticos y corregirlos mediante criterios de fiabilidad contrastada.

La base de datos cubre el periodo temporal 1950-2010 en el que se constata una clara tendencia al incremento térmico, general y con escasas diferencias entre ambas vertientes del Pirineo, de aproximadamente $1,2^{\circ} \mathrm{C}$. El dato es relevante, pero exige mayor desarrollo y sobre todo definir unos indicadores que permitan el análisis de las anomalías y tendencias del clima en el Pirineo. De esa forma, se podrá llevar a cabo un diagnóstico del mismo de forma regular y actualizada. 


\section{Agradecimientos}

Este trabajo se ha realizado en el marco del proyecto de investigación OPCC-POCTEFA EFA 235/11, de la Unión Europea, y del proyecto CGL2011-28255, del Ministerio de Ciencia e Innovación y FEDER. Y ha contado también con la ayuda del Gobierno de Aragón y el Fondo Social Europeo al grupo de investigación consolidado "Clima, Agua y Cambio Global” de la Universidad de Zaragoza.

\section{Bibliografía}

Aguilar, E., Auer, I., Brunet, M., Peterson, T. y Wieringa, J. (2003) Guidelines on climate metadata and homogenization. WCDMPNo.53, WMO-TD No.1186. World Meteorological Organization. Geneve, $51 \mathrm{p}$.

Beck, C., Grieser, J. y Rudolf, B. (2005) A new monthly precipitation climatology for the global landa reas for the period 1951 to 2000. Climate Status Report, 2004. German Meteorological Service: 181-190.

Begert, M., Schlegel, T. y Kirchhofer, W. (2005) Homogeneous temperature and precipitation series of Switzerland from 1864 to 2000. International Journal of Climatology, 25: 65-80.

Böhm, R., Auer, I., Schöner, W., Ganekind, M., Gruber, C., Jurkovic, C., Orlik, A. y Ungersböck, M. (2009) Eine neue Webseite mit instrumentellen Qualitäts Klimadaten für den Grossraum Alpen zurück bis 1760 . Wiener Mitteilungen Band 216: Hochwässer: Bmessung, Risikoanalyse und Vorhersage.

Brunetti, M., Buffoni, L., Maugueri, M. y Nanni, T. (2006) Temperature and precipitation variability in Italy in the last two centuries from homogeneised instrumental time series. International Journal of Climatology, 26: 345-381.

Domonkos, P. (2011) Adapted Caussinus-Mestre Algorithm for homogenising Networks of Temperature series (ACMANT), Int. J. Geosci., 2. 293-309. DOI:10.4236/ijg.2011.23032.
Espejo, F., Ferraz, J. y Palomo, M. (2008) Tendencias recientes en las series de temperatura del Pirineo Central y Occidental. VI Congreso Internacional de la AEC, serie A, 6: 99-108.

Esteban, P., Prohom, M. y Aguilar, E. (2012) Tendencias recientes e índices de cambio climático de la temperatura y la precipitación en Andorra (1935-2008). Pirineos, 167: 71-88.

González Hidalgo, C., Brunetti, M y de Luís, M. (2011) A new tool for monthly precipitation analysis in Spain: MOPREDAS database (monthly precipitation trends December 1945-November 2005). International Journal of Climatology, 31: 715-731.

Guijarro, J.A. (2011) User's guide to CLIMATOL. http://www.meteobal.com/climatol/climatol-guide.pdf

JCR (2010) Climate of the Carpathian Region. Europen Commission Joint Reseach Centre. http://desert.jcr.ec.europa.eu/action/php/in dex.php

Klok, E.J. y Klein Tank, A. (2009) Updated and extended European datast of daily climate observations. International Journal of Climatology, 29: 1182-1191.

Kuglitsch, F.G., Auchmann, R., Bleisch, R., Brönnimann, S., Martius, O. and Stewart, M. (2012) Break detection of annual Swiss temperature series, J. Geophys. Res., 117, D13105, doi: 10.1029/2012JD017729. 
Mestre, O., Domokos, P., Picard, F., Auer, I., Robin, S., Lebarbier, E., Böhm, R., Aguilar, E., Guijarro, J., Vertachnik, G., Klancar, M., Gubuisson, B. and Stepanek, P. (2013) HOMER: a homogenization software - methods and applications. Quart. Jour. of the Hungarian Meteorological Service, 117, 1, pp. 47-67.

Mitchell, T.D. and Jones, P.D. (2005) An improved method of constructing a database of monthly climate observations and associated high-resolution grids. International Journal of Climatology, 25: 693-712.

Peterson, T.C., Easterling, D.R., Karl, T., Groisman, P., Nicholls, N., Plummer, N., Torok, S., Auer, I., Boehm, R., Gullet, D., Vincit, L., Heino, R., Mestre, O., Salinger, J., Forlan, E., Hanssen, I., Alexandersson, H., Jones, J.P. y Parker, D. (1998) Homogeneity adjustments of in situ atmospheric climate data: a review. International Journal of Climatology, 18: 1493-1517.

Picard, F., Lebarbier, E., Hoebeke, M., Rigaill, G., Thiam, B. and Robin, S. (2011) Joint segmentation, calling and normalization of multiple CGH profiles. Biostatistics, 12, pp. 413-428. doi: 10.1093/biostatistics/kxq076.
Soubeyroux, J.M., Jourdain, S., Grimal, D., Espejo, F., Esteban, P. y Merz, T. (2011) Approche transfrontalière pour l'inventaire et la valorization des données climatologiques sur le Massif des Pyrénées. Colloque SHF "Eaux en montagne". Lyon.

Venema, V., Mestre, O., Aguilar, E., Auer, I., Guijarro, J.A., Domonkos, P., Vertacnik, G., Stepanek, P., Zahradnicek, P., Viarre, J., MüllerWestermeier, G., Szentimrey, T., Lakatos, M., Williams, C.N., Menne, M., Lindau, R., Rasol, D., Rustemeier, E., Kolokythas, K., Marinova, T., Andresen, L., Acquaotta, F., Fratianni, S., Cheval, S., Klancar, M., Brunetti, M., Gruber, C., Prohom, M., Likso, T., Esteban, P. and Brandsma, T. (2012) Benchmarking homogenization algorithms for monthly data. Clim. Past Discuss., 8, 89-115. DOI: $10.5194 / \mathrm{cp}-8-89-2012$. 